\title{
NUMERICAL SIMULATION OF SLOSHING IN LNG TANK INCLUDING IRREGULAR TANK MOTION USING MOVING GRID TECHNIQUE
}

\author{
KUNIHIDE OHASHI* \\ ${ }^{*}$ National Maritime Research Institute \\ 6-38-1 Shinkawa, Mitaka, Tokyo, Japan \\ e-mail: k-ohashi@m.mpat.go.jp
}

Key words: Sloshing, Moving Grid, Irregular motion

\begin{abstract}
Numerical simulations of the sloshing phenomena of the liquid inside of a tank with the regular and irregular tank motions are carried out. An in-house structured CFD solver which is capable the moving grid technique is used. The governing equations are 3D Navier-Stokes equations for the incompressible flow. An interface capturing method based on the single phase level-set approach is employed to capture free surface of the liquid. The LNG tank shape which has the experimental data is utilized. The sway motion of the LNG tank is taken account by the grid velocity which is introduced by the amount of the displacement of the computational grid based on the tank motion. First, the regular sway motion is enforced to examine the occurrence of the swirling phenomena which the liquid rotates inside of the tank, and the time histories of the pressure at the positions on the tank wall are validated. Next, the irregular tank motion which is based on the RAO of the ship motion in irregular waves and the power spectrum of the wave is enforced, and the difference of the hydrodynamic forces of the tank between the regular and irregular motions are examined.
\end{abstract}

\section{INTRODUCTION}

Numerical method for the simulation of the sloshing phenomena using the moving grid technique is developed. The tank motion is defined with the regular and irregular motions, and the irregular tank motion is introduced by the power spectrum of the wave and response amplitude operator which is pre-defined. The rectangular LNG tank which has the experimental data[1][2] is selected, and the time histories of the pressure on the tank wall are compared with the measured data at the regular tank motion. The occurrence of the swirling phenomena is also examined. The frequency analysis for the results of the irregular tank motion is performed and the effect of the irregular tank motion is revealed.

\section{COMPUTATIONAL METHOD}

The governing equation is 3D RANS equation for incompressible flows. Artificial compressibility approach is used for the velocity-pressure coupling. For unsteady flow simulations, a dual time stepping approach is used in order to recover incompressibility at each time step.

$$
\frac{\partial \boldsymbol{q}}{\partial t}+\frac{\partial \boldsymbol{q}^{*}}{\partial \tau}+\frac{\partial\left(\boldsymbol{e}-\boldsymbol{e}^{v}\right)}{\partial x}+\frac{\partial\left(\boldsymbol{f}-\boldsymbol{f}^{v}\right)}{\partial y}+\frac{\partial\left(\boldsymbol{g}-\boldsymbol{g}^{v}\right)}{\partial z}=0
$$




$$
\begin{gathered}
\boldsymbol{q}=\left[\begin{array}{llll}
0 & u & v & w
\end{array}\right]^{T}, \quad \boldsymbol{q} *=\left[\begin{array}{cccc}
p & u & v & w
\end{array}\right]^{T} \\
\boldsymbol{e}=\left[\begin{array}{c}
\beta u \\
\left(u-u_{g}\right) u+p \\
\left(u-u_{g}\right) v \\
\left(u-u_{g}\right) w
\end{array}\right], \boldsymbol{f}=\left[\begin{array}{c}
\beta v \\
\left(v-v_{g}\right) u \\
\left(v-v_{g}\right) v+p \\
\left(v-v_{g}\right) w
\end{array}\right], \boldsymbol{g}=\left[\begin{array}{c}
\beta w \\
\left(w-w_{g}\right) u \\
\left(w-w_{g}\right) v \\
\left(w-w_{g}\right) w+p
\end{array}\right], \\
\boldsymbol{e}^{v}=\left[\begin{array}{c}
0 \\
\tau_{x x} \\
\tau_{x y} \\
\tau_{z x}
\end{array}\right], \boldsymbol{f}^{v}=\left[\begin{array}{c}
0 \\
\tau_{x y} \\
\tau_{y y} \\
\tau_{y z}
\end{array}\right], \boldsymbol{g}^{v}=\left[\begin{array}{c}
0 \\
\tau_{z x} \\
\tau_{y z} \\
\tau_{z z}
\end{array}\right]
\end{gathered}
$$

where $\left(u_{g}, v_{g}, w_{g}\right)$ is grid velocities due to the moving grid, $\beta$ is parameter of the artificial compressibility approach and $\beta=1.0$ is given in the present computation. $\tau_{i j}$ is defined as $\tau_{i j}=\frac{1}{R}\left(\frac{\partial u_{i}}{\partial x_{j}}+\frac{\partial u_{j}}{\partial x_{i}}\right), R$ is Reynolds number, $v$ is kinematic viscosity coefficient.

An in-house structured CFD solver [3] is employed. The governing equation is 3D RANS equation for incompressible flows. Artificial compressibility approach is used for the velocity-pressure coupling. Spatial discretization is based on a finite-volume method. A cell centered layout is adopted in which flow variables are defined at the centroid of each cell and a control volume is a cell itself. Inviscid fluxes are evaluated by the third-order upwind scheme based on the flux-difference splitting of Roe. The evaluation of viscous fluxes is second-order accurate. For unsteady flow simulations, the dual time

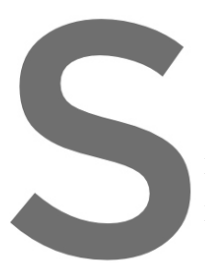
stepping approach is use second order two-step bac scheme for the pseudo tim method. For free surface is employed.
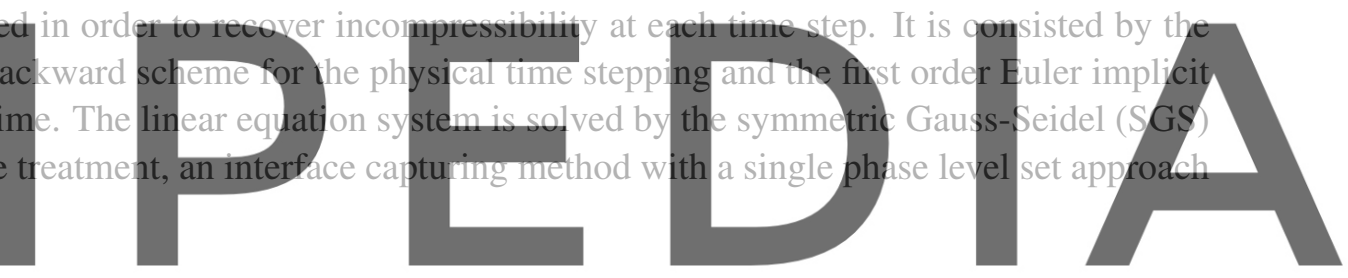

3 COMPUTED RESULTS

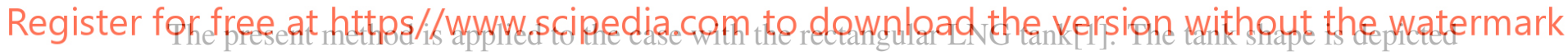

in Figure 1, and the width becomes narrower near the top and bottom of the tank. The details of the tank shape can be found in [1], the tank length is $0.971 \mathrm{~m}$, tank width is changed 0.93 and 1.02 based on the tank width and length ratio. The time histories of the pressure at the positions $P_{1}$ and $P_{2}$ are compared with the measured data. The position $P_{1}$ locates over the static liquid level, instead the position $P_{2}$ locates always under the liquid level. The liquid level is given as $50 \%$ of the tank height.

Table 1 shows the division number of the computational grid, IM means the longitudinal division number, $\mathrm{JM}$ is lateral direction, $\mathrm{KM}$ means the division number in the height direction. The two computational grids $G_{1}$ and $G_{2}$ are utilized to examine the effect of the grid division number.

Table 1: Division number of computational grid

\begin{tabular}{cc}
\hline Grid & $\mathrm{IM} \times \mathrm{JM} \times \mathrm{KM}$ \\
\hline$G_{1}$ & $37 \times 73 \times 29$ \\
\hline$G_{2}$ & $73 \times 145 \times 57$ \\
\hline
\end{tabular}




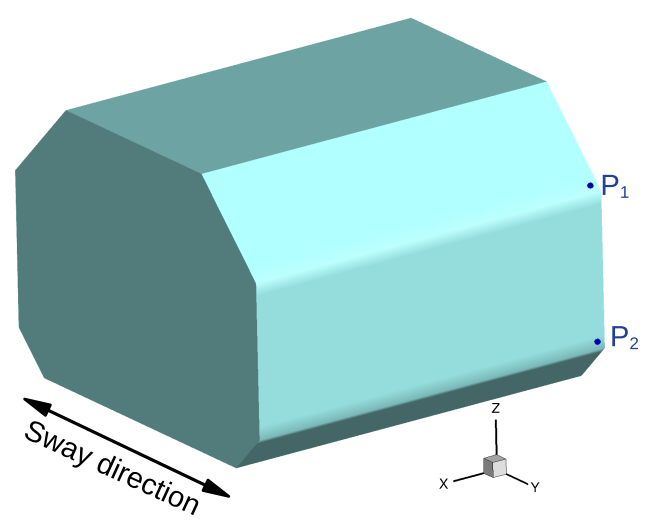

Figure 1: Overview of LNG tank shape

\subsection{Sloshing in regular motion}

The tank moves in y-direction with the sway motion. The excitation frequency is set as $f=0.804 \mathrm{~Hz}$ and the one sided amplitude $0.02 \mathrm{~m}$ which are determined by the encounter sea state and ship response amplitude at the sea state condition. Figure 2 shows the time histories of the pressure at the points $P_{1}$ and $P_{2}$ under the sloshing condition. The impact pressure can be found in the time history at $P_{1}$. The present computation results show agreement with the measured data. The results with the grid $G_{2}$ shows better

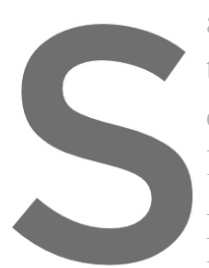
agreement comparing

the impact point, then rise of the pressure at $P_{2}$ shows level, consequently, the in Figure 3 shows the time histories of the hydrodynan longitudinal and lateral directions with the the tank
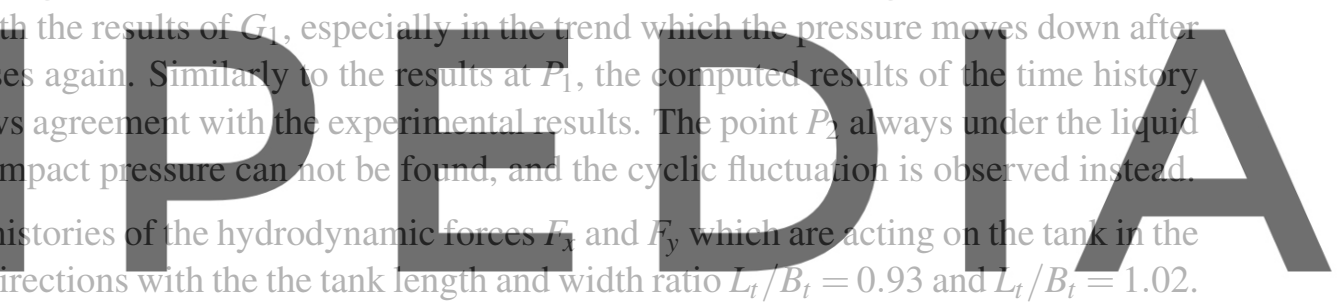

The hydrodynamic forces are non-dimensionalized by $\rho g L_{t} B_{t} h$ where $h$ means the liquid height in the

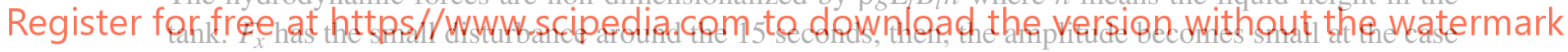

with $L_{t} / B_{t}=1.02$. $F_{y}$ has the large fluctuation with the cyclic period. On the other hand, $F_{x}$ has the

fluctuation which is similar order with the amplitude of $F_{y}$ at the case with $L_{t} / B_{t}=0.93$. The swirling phenomena can be observed in this case. The period of $F_{x}$ has the difference in the quarter period comparing with the time history of $F_{y}$.

Figure 4 shows the instantaneous view of free surface of the liquid in the tank with the tank length and width ratio $L_{t} / B_{t}=0.93$ and $L_{t} / B_{t}=1.02$. The instantaneous view of free surface takes the two dimensional shape in the longitudinal direction which can be observed in the sloshing phenomena at $L_{t} / B_{t}=1.02$. The liquid rotates in the tank at the case with $L_{t} / B_{t}=0.93$. The liquid surface rises at the tank longitudinal end wall when $F_{x}$ becomes larger in the time history in Figure 3, then, the liquid surface rises at the one side wall and becomes lower at the opposite side wall when $F_{y}$ takes high value. The present method reveals the swirling phenomena and captures the interaction between the tank wall and the liquid inside of the tank. 

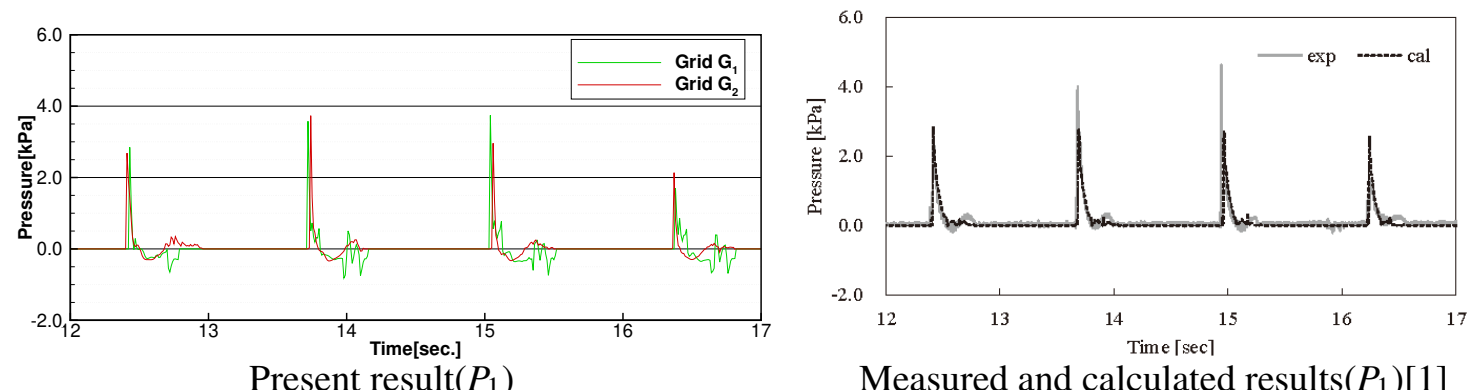

Measured and calculated results $\left(P_{1}\right)[1]$
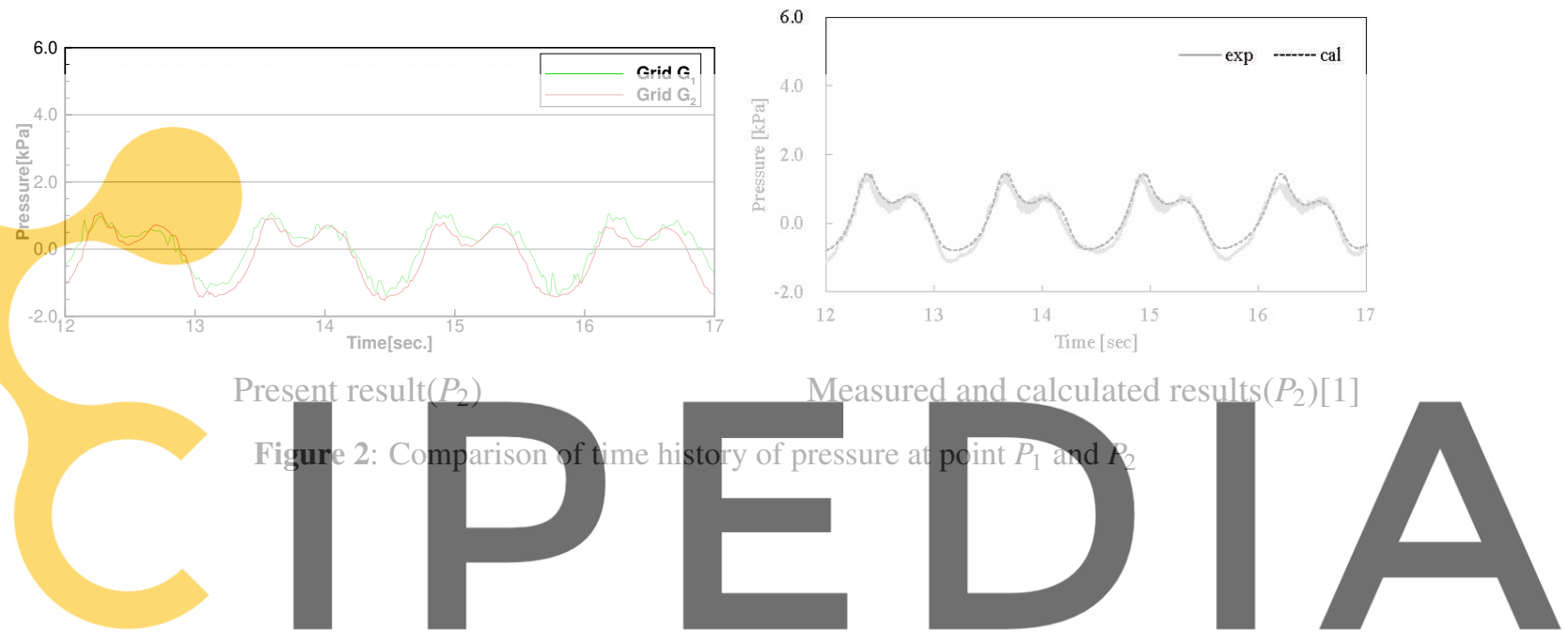

Register for free at https//www.scipedia.com to download the version without the watermark
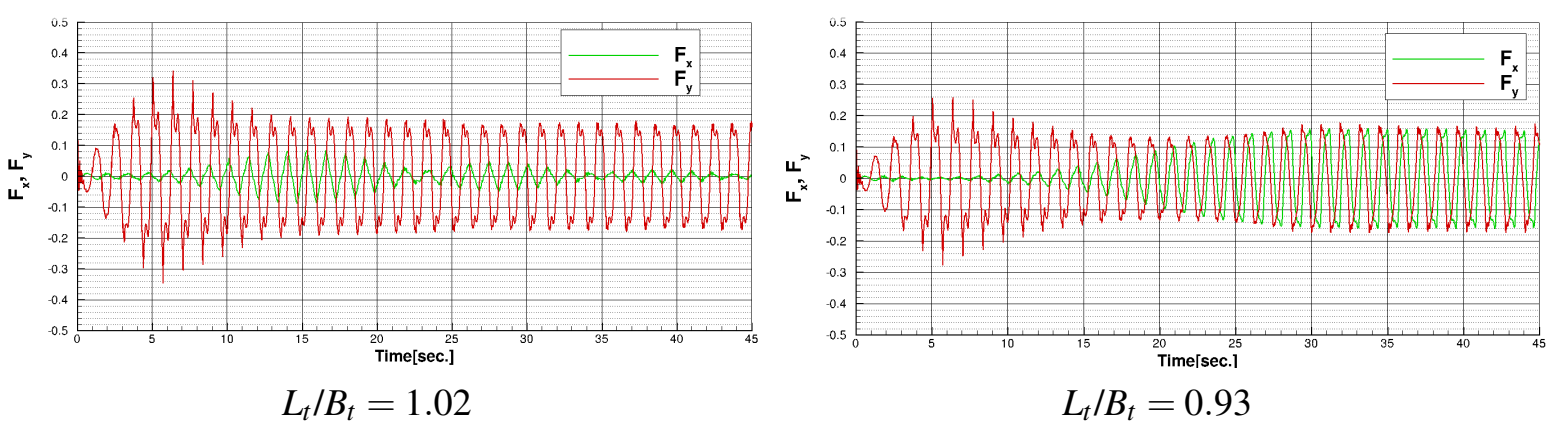

Figure 3: Time history of force $F_{x}$ and $F_{y}$ 


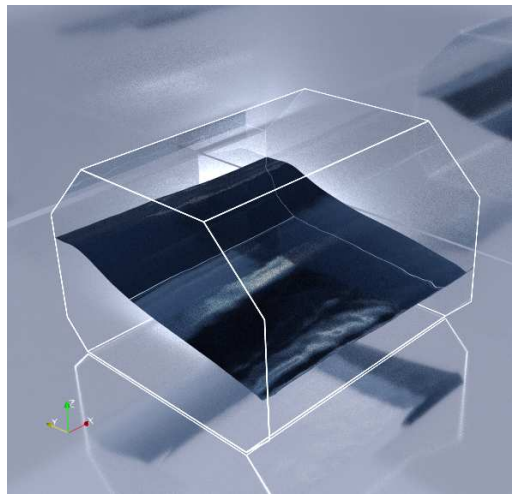

$L_{t} / B_{t}=1.02$

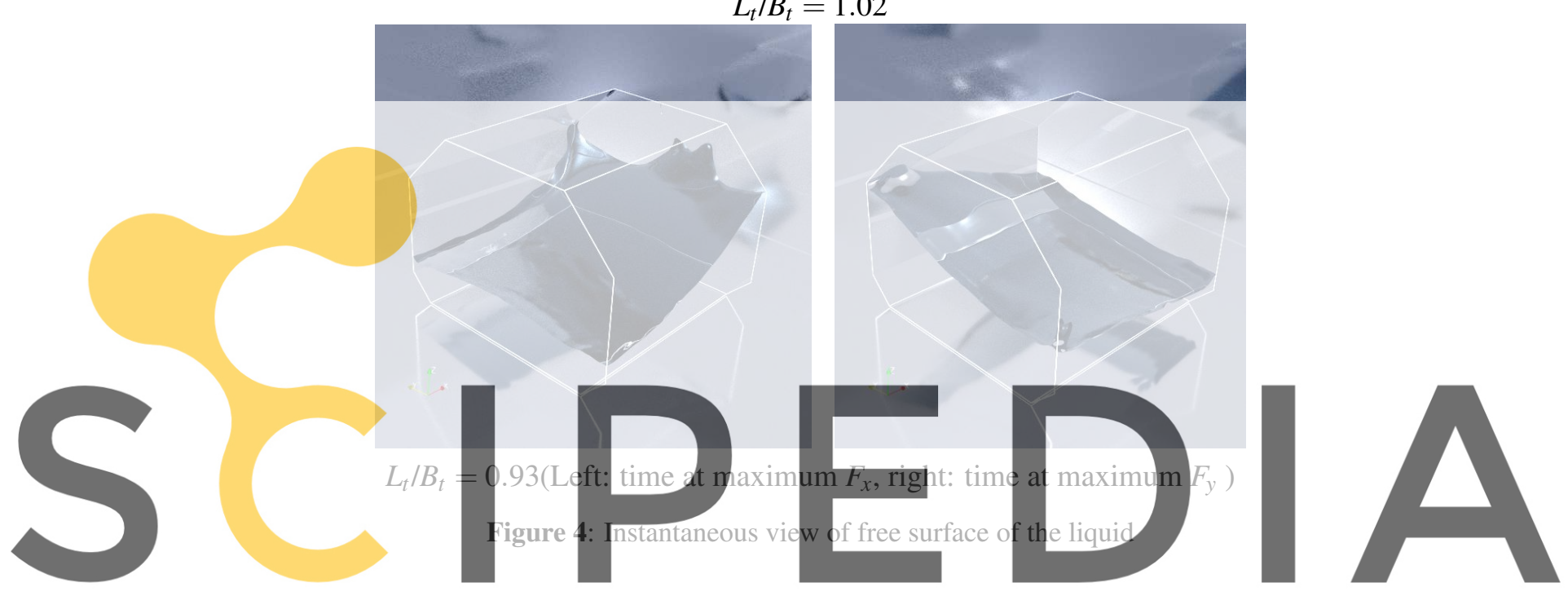

3.2 Sloshing in irregular motion

Register for free at https/fww.w. scipedia,com to download the version without the watermark

are same as the regular motion case with the the tank length and width ratio $L_{t} / B_{t}=1.02$, and the grid

$G_{2}$ is utilized. The tank irregular motion can be obtained by the frequency spectrum and the response amplitude operator which is pre-defined. The modified Pierson-Moskowitz spectrum[4][5] is given by the following equation with the $1 / 3$ significant wave height $H_{1 / 3}$, the averaged wave period $T_{a v e}$ and the frequency $\omega$ as follows:

$$
S(\omega)=\frac{A_{f}}{\omega^{-5}} \exp \left(-\frac{B_{f}}{\omega^{4}}\right)
$$

where $A_{f}=173 H_{1 / 3}^{2} / T_{a v e}^{4}, B_{f}=691 / T_{a v e}^{4}$. The tank irregular motion can be obtained by the response amplitude operator in the similar way in the reference[2]. Figure 5 shows the response amplitude operator based on the non-dimensionalized frequency. Once, the wave half amplitude $\sqrt{2 S(\omega) \Delta \omega}$ is obtained by the frequency spectrum, then, the tank motion is introduced by the integration of the response amplitude operator using the frequency and wave amplitude in the each times step. $\Delta \omega$ means the division width in the frequency range, and the effect of $\Delta \omega$ is also examined. 


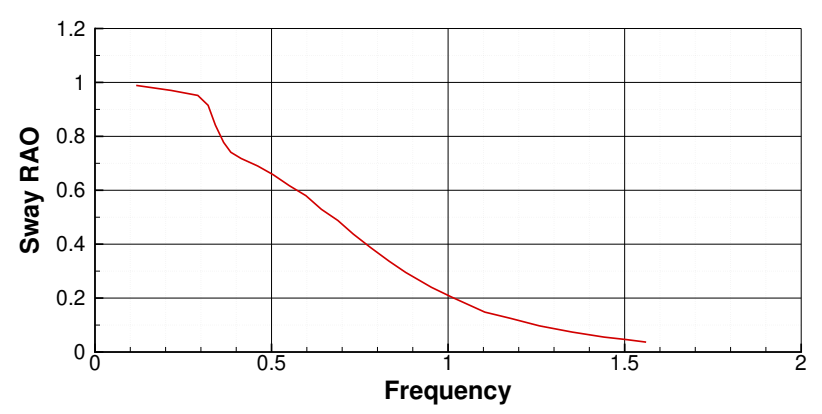

Figure 5: Response amplitude operator of sway motion

The sea state condition is defined with the $H_{1 / 3}=5.76 \mathrm{~m}$ and $T_{\text {ave }}=9.55 \mathrm{sec}$. at the equivalent to the actual condition. The frequency range is given with $f_{\min }=0.195$ and $f_{\max }=1.56$ in Figure 5. The non-dimensionalized time step Deltat $=0.01$ which divides the averaged period $T_{\text {ave }}$ with 150 and the wave period at $f_{\max }=1.56$ with 65 . The simulation is carried out in approximately 1300 seconds in the actual condition.

The effect of $\Delta \omega$ is examined with changing the division number 100, 150, 200. Figure 6 shows the comparison of the time history of the tank motion. All the division numbers give the irregular motion, and Figure 7 shows the results of the frequency analysis with the target line. All the computed results show the deviation around the target line, and the result with the division number 200 shows the smaller

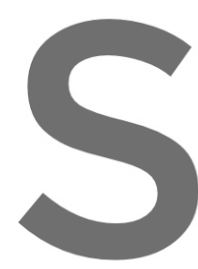
deviation comparing with further analysis is performed. Figure 8 shows the time history ation. The longitudinal force $C$ not observed in this condition. Figure
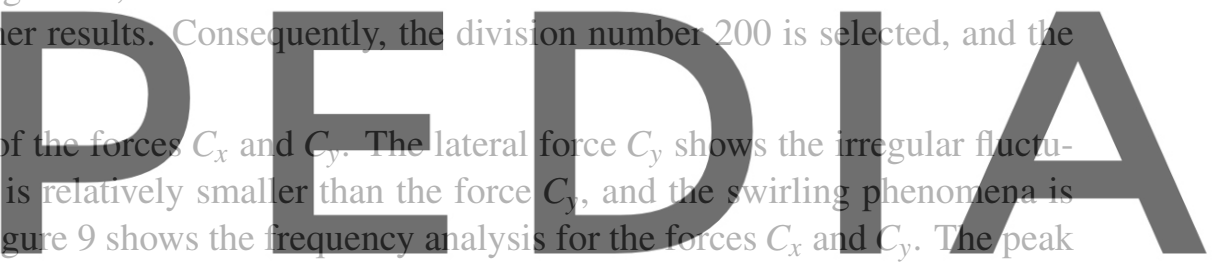

frequency is approximately 0.76 and differs from the wave frequency which is introduced by the aver-

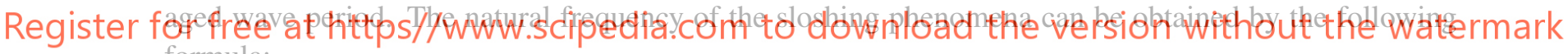
formula:

$$
\left.f_{n}=\frac{1}{2 \pi} \sqrt{(} \frac{g \pi}{B_{t}} \tanh \frac{\pi h}{B_{t}}\right)
$$

The natural frequency of the sloshing phenomena is approximately 0.79 which is close to to the peak frequency of the forces $C_{x}$ and $C_{y}$. The peak frequency of the forces $C_{y}$ with $L_{t} / B_{t}=1.02$ in Figure 3 is approximately 0.75 , thus, the hydrodynamic forces takes the peak value at the natural frequency of the sloshing phenomena. The force $C_{y}$ takes third order of the natural frequency which is derived from from the tank shape.

Figure 10 shows the time history of the pressure at the position $P_{1}$ and $P_{2}$ on the irregular tank motion. The impact pressure can be found at $P_{1}$, and the order of the pressure value is similar with the results of the regular tank motion. The irregular fluctuation can be observed at $P_{2}$, and the results of frequency analysis is shown in Figure 11. The pressure also takes the peak value at the natural frequencies of the sloshing phenomena, and the second order of the natural frequency can be found. 

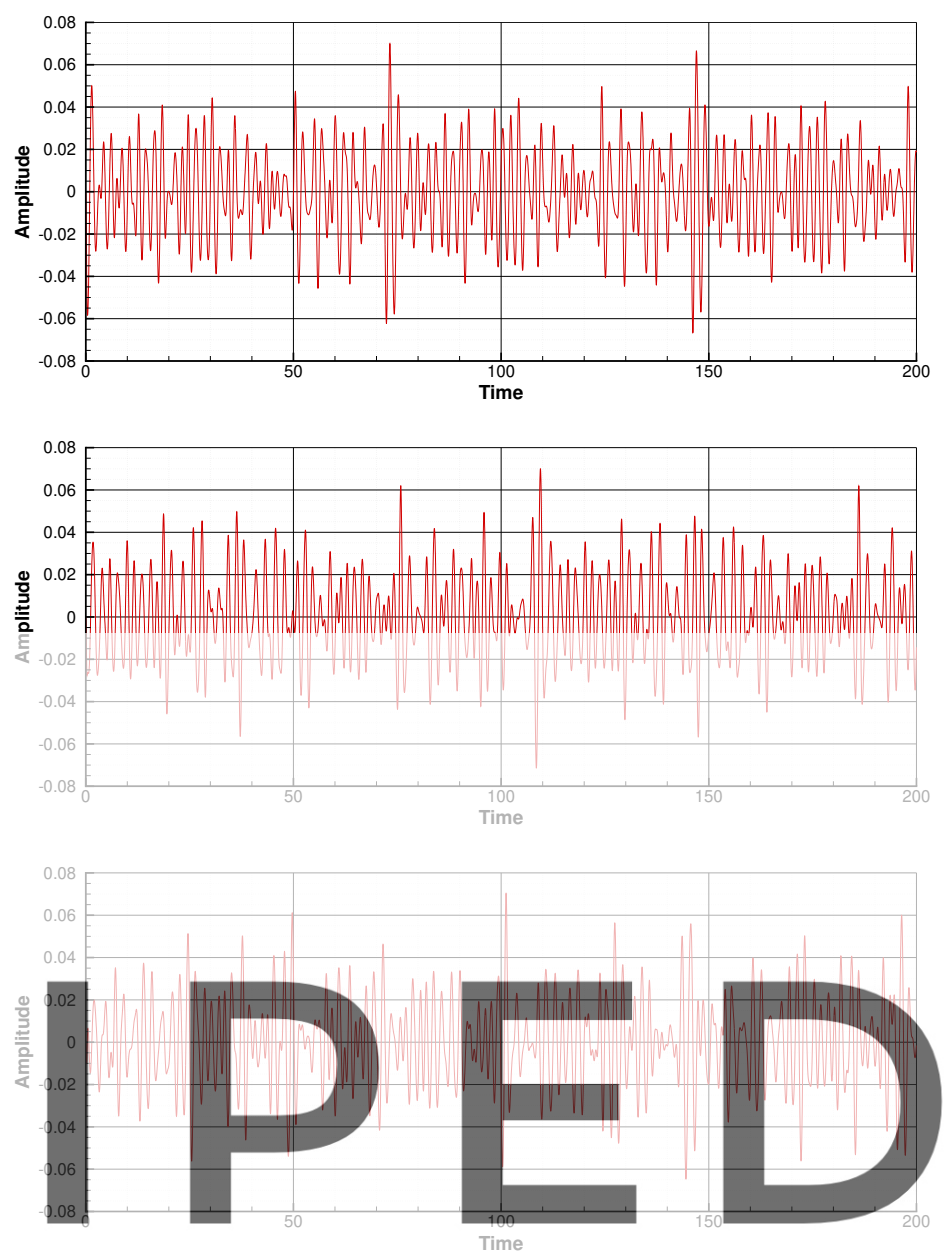

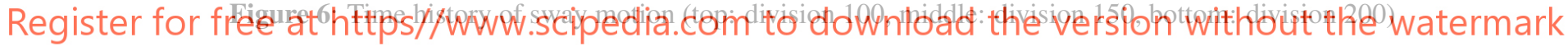

\section{CONCLUSIONS}

- The numerical method to simulate the sloshing phenomena with the regular and irregular tank motions using the moving grid technique is developed.

- The computed results of the time histories of the pressure on the tank wall is compared with the measured results, and the present results show agreement with the existed experimental data.

- The swirling phenomena can be observed in the regular tank motion.

- The hydrodynamic forces and pressure on the tank wall take the peak value at the natural frequencies of the sloshing phenomena in regular and irregular motions.

\section{ACKNOWLEDGEMENT}

This work has been supported by JSPS KAKENHI Grant Number JP19K04869. 

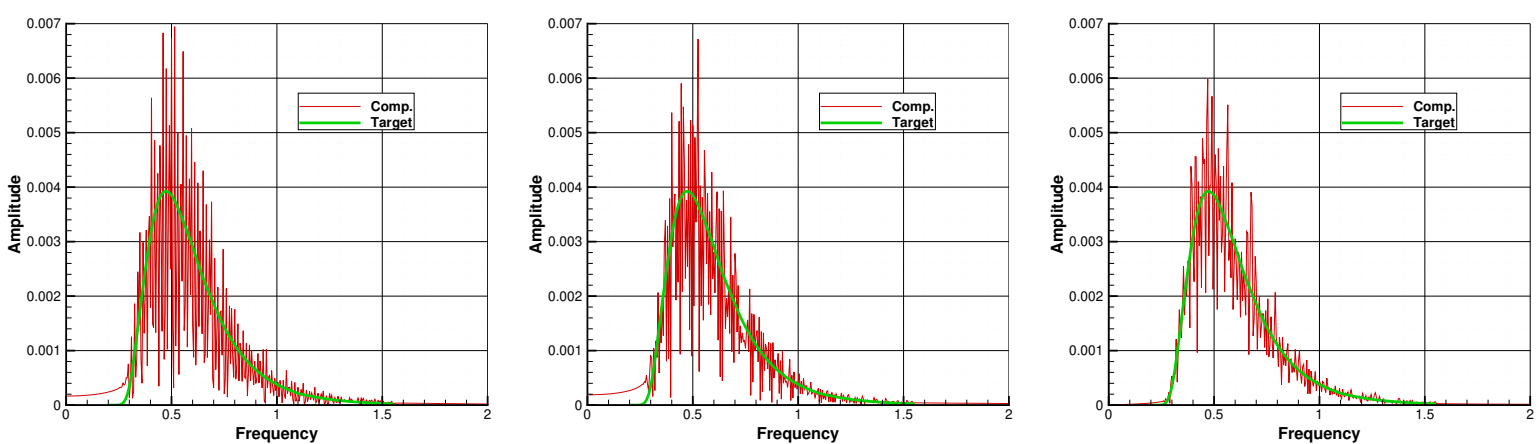

Figure 7: Results of frequency analysis (left: division 100, middle: division 150, right: division 200)
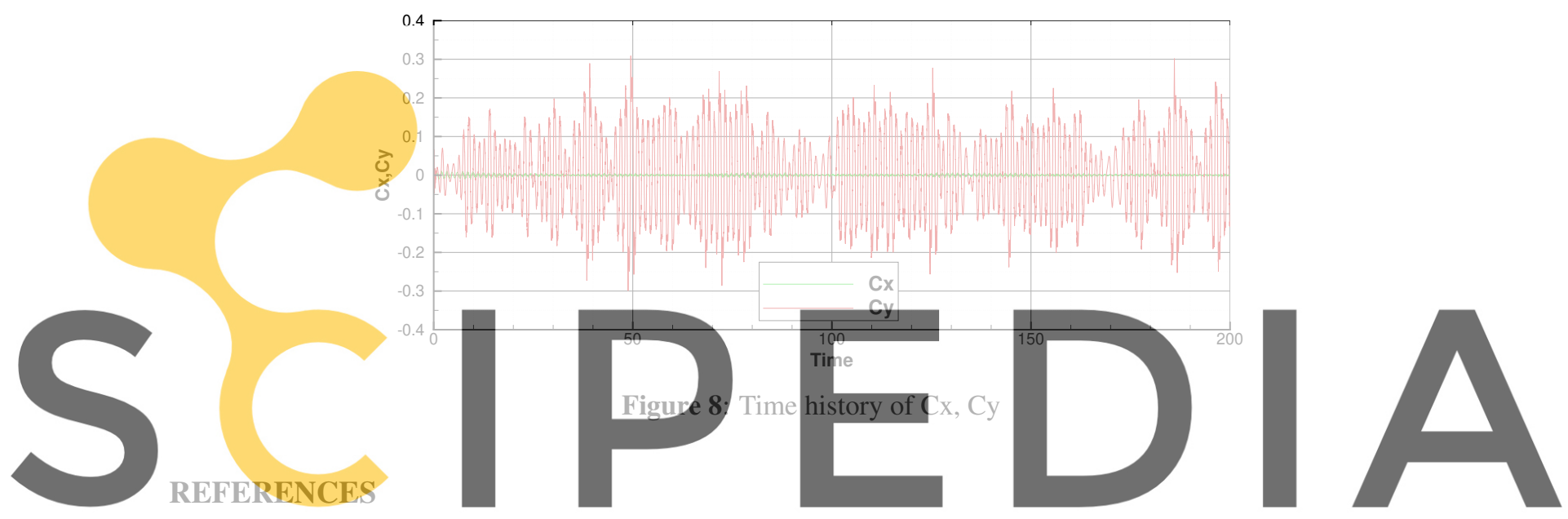

[1] Arai, M. et al. Experimental and numerical study of sloshing and swirling in partially filled

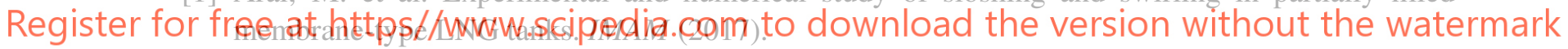

[2] Yoshida, T. et al. Model experiments and numerical simulations of sloshing and swirling in membrane LNG tanks. J. of the JASNAOE (2018) 27 (in Japanese)

[3] Ohashi, K. et al. Development of a structured overset Navier-Stokes solver with a moving grid and full multigrid method. J. Mar. Sci. Tech. (2019) 24:884-901.

[4] ITTC recommended procedures and guidelines 7.5-04-01-01.2, analysis of speed/power trial data.

[5] Final report and recommendations of the specialist committee on waves, 23rd ITTC. 

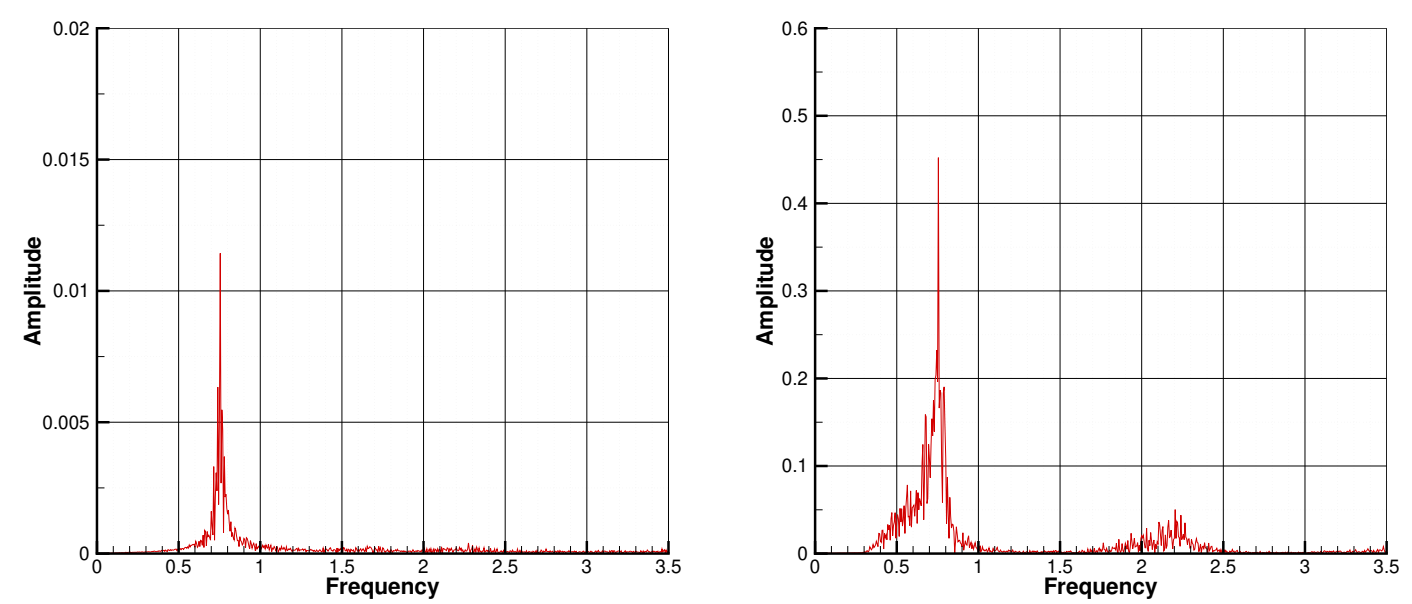

Figure 9: Results of frequency analysis (left: $C_{x}$ right: $C_{y}$ )
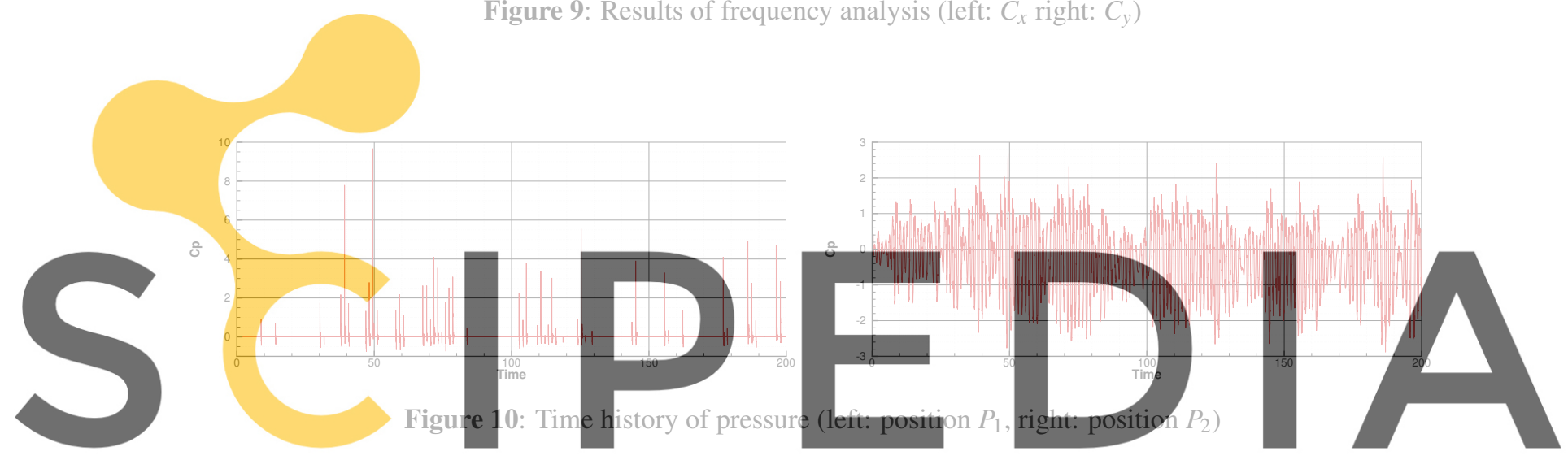

Register for free at https//www.scipedia.com to download the version without the watermark

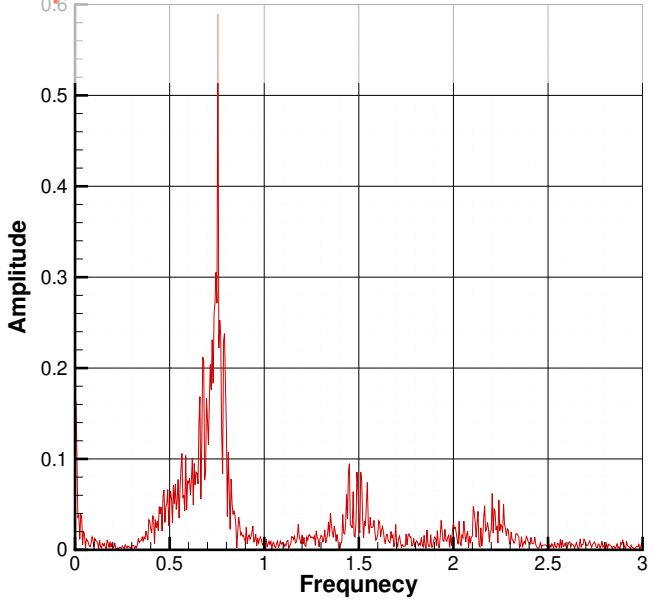

Figure 11: Results of frequency analysis on $P_{2}$ 